meeting was followed in August of 2005 by a small meeting in Portland, Oregon focused on research opportu-

\title{
The Tobacco Research Network on Disparities (TReND)
}

\section{Richard R Clayton}

\section{The mission of TReND is to eliminate tobacco related disparities through transdiscipliniary research that advocates the science, translates this scientific knowledge into practice, and informs public policy.}

T here are two important goals for the USA in Healthy People 2010. The first is to increase quality and years of healthy life. The second is to eliminate health disparities. These disparities include differences in tobacco related outcomes as well as patterns of tobacco use by gender, race/ethnicity, socioeconomic status and socioeconomic position (that is, education, income, occupational status, wealth), geographical location, sexual orientation, and various forms of disability. The 2000 surgeon general's report Reducing Tobacco Use identified eliminating health disparities related to tobacco use as a major public health challenge. In 2002 representatives from a number of disciplines and organisations met at a National Conference on Tobacco and Health Disparities. In addition to reviewing the existing science and highlighting gaps in the knowledge base, one outcome of this conference was development and articulation of a research agenda for eliminating tobacco related health disparities. ${ }^{1}$

Another important outcome of this conference was explicit recognition that tobacco related health disparities are very complex and exist at many levels, thus requiring a transdisciplinary perspective. ${ }^{2-5}$ This led the National Cancer Institute, in collaboration with the American Legacy Foundation to establish the Tobacco Research Network on Disparities (TReND).

After a period of strategic planning, 21 scientists representing a variety of academic disciplines were invited to become core members of this transdisciplinary research network. These scientists work at 11 major research universities, two NIH Institutes, two philanthropic foundations, and one HBCU (historically black college and university) (the details of the core members of the network are listed at the end of the article).

The mission of TReND is to eliminate tobacco related disparities through transdisciplinary research that advances the science, translates that scientific knowledge into practice, and informs public policy. While there has been some progress made in reporting tobacco related disparities, many questions remain about the epidemiology and aetiology of these disparities, and the amount of heterogeneity that exists between and within various population groups. Moreover, little is known about the ways in which differences in tobacco use patterns and norms, and their effects, lead to disparities in health and other outcomes. The operating principles of TReND are: transdisciplinarity, stimulation and integration of disparities research, promotion of disparities research, translation of knowledge about tobacco related disparities into practice, and a commitment to diversity.

The goals of TReND are to: (1) generate new ideas and research projects focusing on tobacco and health disparities; (2) encourage collaborations among researchers representing multiple research disciplines; (3) establish mechanisms to effectively translate the science, communicate the results, and interact with other networks and community advocacy groups; and (4) promote the development of junior investigators and the participation of senior researchers in health disparities research.

The entire network has met a total of eight times to this point in time (June 2006). In most of these meetings there has been a focus on tobacco related disparities in particular population groups. For example, in the February 2005 meeting in Pasadena there were presentations and discussions about tobacco among Asian/Pacific Islanders and the LGBT communities. In May/ June of 2005 TReND met in Anchorage, Alaska with a number of representatives from the Alaska Native Tribal Health Consortium concerning culturally specific tobacco use patterns and consequences among Alaska Natives. This nities and challenges in Alaska. In December of 2005, TReND met in Austin, Texas with representatives from the National Latino Cancer Research Network with a special focus on the so called Hispanic paradox. In June of 2006 the network met with representatives of the University of Oklahoma Community Network Project involving two American Indian groups (the Choctaw and the Cherokee Nations) and representatives from the Conference of Black Mayors representing leaders from Oklahoma towns whose populations are predominantly African American.

In addition to the focus on specific population groups, there has been a substantial amount of discussion and thought devoted to the conceptualisation and measurement of socioeconomic status (SES) and position. On a number of occasions the question has arisen about whether there is a need for a $21 \mathrm{st}$ century conceptualisation and measurement of SES; the most widely used measures and parameters of SES have not changed much in the past half century. In line with these observations, the network sponsored a small, one day conference to begin exploring how to approach such an iconic construct in comprehensive and constructive ways.

The network is involved in a number of activities directed at expanding the scientific knowledge base about tobacco related health disparities. One of the most important of these activities was the September 2005 conference on tobacco policies and low SES women and girls, the stimulus for the outstanding papers appearing in this issue. Another of these activities will occur in July of 2006. Under the auspices of NCI, the network is hosting a one day health disparities research methods training symposium in Washington immediately before the world conference on tobacco OR health. The papers to be presented at this meeting review state of the science methodological, design, and statistical approaches to understanding health disparities. A complementary purpose of the meeting is networking among health disparities researchers who applied for the limited number of participant slots at the meeting. Another activity of TReND currently in the planning stages is a meeting that will occur in the autumn of 2006. That meeting will focus on methodological (particularly sampling) and substantive issues associated with generating estimates of tobacco use and its consequences among population groups that are relatively small in size and largely invisible. The specific focus of this 
meeting and the products that will come from it will be on tobacco use among LGBT populations of colour.

The network also has a series of papers that are under review for a special supplemental issue of Addiction. This issue will comprehensively review conceptual and methodological issues concerning tobacco related health disparities. It is expected that this issue will be published sometime in 2007. At the present time the network is involved in planning a study that will entail developing new measures of media exposure, validating them in a wide range of communities in which specific population groups are the majority, and inserting them in a national evaluation of a media campaign that will be implemented in 2007. This network activity will involve research collaboration with several of NCI's Community Cancer Networks and an attempt to better understand the role of exposure to various ethnic media and their influence on behaviour change regarding tobacco.

Transdisciplinary research networks are challenging for a number of reasons. The first is the novelty of a large group of scientists, many of whom had never met before becoming a member of the core group, establishing rapport and trust when everyone is in the same room for only a small number of occasions each year. To this is added the challenge of learning how to interact and communicate across the disciplinary language, methodological, and conceptual barriers that exist. The approach taken by TReND has been to establish working groups composed of network members and participants from outside the network with special expertise. These working groups are focused on specific topics and tasks like those described above. Much of the work is performed with conference calls. For example, from 1 May 2005 to 1 May
2006, the network had a total of 128 conference calls by working groups that entailed over 800 person hours.

The papers in this special issue of the Journal of Epidemiology and Community Health provide an excellent example of this activity. The network identified an important gap in the knowledge base concerning the effects of tobacco control policies on low SES women and girls. The TReND working group led by Deborah McLellan and Nancy Kaufman planned the meeting held 22-23 September 2005 in Bethesda on "Tobacco control policies: Do they make a difference for low SES women and Girls?" A more in depth description of the process and the meeting can be seen in the commentary by McLellan and Kaufman in this issue.

The elimination of tobacco related health disparities will require these kinds of concerted efforts bringing the science and the practice together to understand how public policies influence the lives and health of disparate population groups. We applaud the intellectual rigour of the authors of these papers and acknowledge with gratitude and respect, the incredible amount of effort expended by the editors of this report, Lorraine Greaves, Donna Vallone, and Wayne Velicer. The members of TReND hope that everyone interested in tobacco related health disparities will be inspired by these excellent research papers to renew their commitment to the elimination of these disparities.

J Epidemiol Community Health

2006;60(Suppl II):ii3-ii4.

doi: $10.1136 /$ jech.2006.052472

Correspondence to: Dr R R Clayton, University of Kentucky College of Public Health, 121 Washington Avenue Suite 110 Lexington, KY 40536-0003, USA; clayton@uky.edu

Funding: the Tobacco Research Network on Disparities is funded by the National Cancer
Institute (NCI) and the American Legacy Foundation.

Competing interests: none.

Core members of TReND: Brian Flaherty, University of Washington; Robert Bendel, Washington State University; Eliseo PerezStable, University of California San Francisco; Lourdes Baezconde-Garbanati, University of Southern California; Dennis Trinidad, University of California San Diego; Gillian Barclay, Kellogg Foundation; Sherry Emery, University of Illinois Chicago; Laura Beebe, University of Oklahoma; George Hammons, Philander Smith College; Mark Hayward, University of Texas; Wayne Velicer, University of Rhode Island; Jennifer Doucet, University of Rhode Island; Vish Viswanath, Harvard University and Dana Farber Cancer Center Harvard; Deborah McLellan, Harvard University and Dana Farber Cancer Center; Eric Moolchan, National Institute on Drug Abused; Vickie Shavers, National Cancer Institute; Pebbles Fagan, National Cancer Institute; Deirdre Lawrence, National Cancer Institute; Donna Vallone, American Legacy Foundation; Anita Fernander, University of Kentucky; Linda Alexander, University of Kentucky; Richard Clayton, University of Kentucky.

\section{REFERENCES}

1 Fagan $P$, King G, Lawrence D, et al. Eliminating tobacco-related health disparities: directions for future research. Am J Public Health 2004;94:211-17.

2 Abrams DB, Clayłon RR. Transdisciplinary research to improve brief interventions for addictive behaviors. In: Monti PM, Colby SM $\mathrm{O}^{\prime}$ Leary TA, eds. Adolescents, alcohol, and substance abuse: reaching teens through brief interventions. New York: The Guilford Press, 2001

3 Clayton RR, Merikangas KR, Abrams DB. Introduction to tobacco, nicotine, and youth: the Tobacco Etiology Research Network. Drug Alcohol Depend 2000;59(suppl 1):S1-4.

4 Clayton RR, Prager DP. Robert Wood Johnson Foundation, Tobacco Etiology Research Network (TERN): grant results. 2005. http:// www.rwjf.org/portfolios/resources/ grantsreport.jsp?filename $=$ tern. htm \&iaid $=143$ grantsreport.jsp?filename

5 Glass TA, McAtee MJ. Behavioral science at the crossroads in public health: extending the horizons, envisioning the future. Soc Sci Med 2006:62:1650-71. 